\title{
ARTICLE Drug-taking in a socio-sexual context enhances vulnerability for addiction in male rats
}

\author{
Lindsey B. Kuiper ${ }^{1}{ }^{1}$, Lauren N. Beloate ${ }^{1}$, Braxton M. Dupuy ${ }^{1}$ and Lique M. Coolen (iD ${ }^{1,2}$
}

Vulnerability to develop addiction is influenced by numerous factors, including social behavior. Specifically, in human users, drug taking in a socio-sexual context appears to enhance further drug-seeking behavior. Users report heightened sexual pleasure as a motivation for further drug use and display risk behaviors even when tested in drug-free state. Here, using a preclinical model of limited voluntary drug use in rats, the hypothesis was tested that methamphetamine (Meth)-taking concurrently with socio-sexual experience increases vulnerability to addiction. Male Sprague Dawley rats were socially housed and underwent limited-access Meth self-administration (maximum $1 \mathrm{mg} / \mathrm{kg} / \mathrm{session).} \mathrm{Meth-taking} \mathrm{was} \mathrm{either} \mathrm{concurrent} \mathrm{or} \mathrm{non-concurrent} \mathrm{with} \mathrm{sexual} \mathrm{behavior:}$ concurrent animals were mated with a receptive female immediately after each session, while non-concurrent animals gained equivalent sexual experience the week prior. Next, drug-seeking behaviors were measured during cue reactivity, extinction, and reinstatement sessions using different extinction and reinstatement protocols in 4 separate studies. Both groups equally acquired Meth self-administration and did not differ in total Meth intake. However, drug-seeking behavior was significantly higher in concurrent animals during cue reactivity tasks, extinction sessions, and cue- or Meth-induced reinstatement tests. In addition, sexual behavior in the absence of Meth triggered reinstatement of drug-seeking in concurrent animals. These results indicate that Meth-taking in a socio-sexual context significantly enhances vulnerability for drug addiction in male rats. This preclinical paradigm of drug self-administration concurrent with socio-sexual behavior provides a useful model for studying the underlying neurobiology of socially driven vulnerability to drug addiction.

Neuropsychopharmacology (2019) 44:503-513; https://doi.org/10.1038/s41386-018-0235-1

\section{INTRODUCTION}

Drug addiction, or substance use disorder, is a major health concern affecting people worldwide, and is characterized by maladaptive drug-seeking and taking despite known negative consequences and life-long risk for relapse to drug taking [1]. Vulnerability to develop addiction is influenced by social and other natural reward-related factors [2-4] and there is increased awareness that drug addiction may share common neural mechanisms with the maladaptive pursuit of non-drug rewards [5-9], i.e., "shared brain vulnerabilities" [5]. Furthermore, drug addiction is often co-morbid with the maladaptive pursuit of sexual reward, i.e., hypersexuality $[6,8,10]$. This association between drug abuse and sexual risk behavior is independent of gender or sexual orientation $[11,12]$ and has been reported for many drugs of abuse [3, 12-16], including methamphetamine (Meth). Drug users report heightened sexual desire, arousal, and pleasure [17] and identify these as a primary motivation for drug use [18]. Together, these facts form the basis for the hypothesis that the association between drug use and sexual behavior leads to increased vulnerability to develop both drug addiction and hypersexuality. However, reports in humans are largely anecdotal and this hypothesis remains to be tested directly and systematically using preclinical models. We previously demonstrated in male rats that Meth administration, when followed immediately by mating behavior (here termed concurrent Meth and sex) resulted in maladaptive sex-seeking behavior or hypersexuality [19-21]. The current study directly tests the hypothesis that Meth taken concurrently with sexual behavior will increase the vulnerability to develop drug addiction in male rats.

Using a conditioned place preference (CPP) paradigm, we previously demonstrated that concurrent Meth and sex increased meth-associated reward-seeking behavior, while diminishing reward for mating when it is experienced without Meth [20]. Even though drug-induced CPP is a very useful and reliable measure for drug-seeking behavior [22], instrumental tasks for drug-seeking behavior are advantageous, and may better reflect key elements of human drug-taking and drug-seeking behaviors, and thus, are considered to be more relevant to the human condition [23]. Therefore, to test the hypothesis that Meth-taking in the context of socio-sexual behavior increases subsequent drug-seeking behaviors, operant self-administration procedures were used. In order to best model the initial recreational drug use in humans typically associated with use of lower doses at longer inter-administration intervals [24], animals were provided restricted access to Meth and were allowed a total intake of only $1 \mathrm{mg} / \mathrm{kg} / \mathrm{session}$ of Meth and a low number of daily sessions. In rats, this Meth dose does not affect sexual performance or motivation but leads to hypersexuality when passively administered concurrently with sexual behavior [19-21]. Hence, the goal of the current set of studies was to determine the specific

\footnotetext{
${ }^{1}$ Department of Neurobiology and Anatomical Sciences, University of Mississippi Medical Center, Jackson, Mississippi, USA and ${ }^{2}$ Department of Physiology and Biophysics, University of Mississippi Medical Center, Jackson, Mississippi, USA

Correspondence: Lique M. Coolen (Icoolen@kent.edu)
}

Received: 29 August 2018 Revised: 12 September 2018 Accepted: 30 September 2018

Published online: 6 October 2018 
contribution of drug taking experienced concurrently with sociosexual behavior to subsequent drug-seeking in groups with equal and restricted drug-taking experience, utilizing various procedures for extinction and relapse (i.e., reinstatement) of drug-seeking [23, 25]. It was previously shown that animals with prior exposure to concurrent Meth and sex do not form a CPP for mating alone, indicating reduced sexual reward when experienced without concurrent Meth [20]. Using a modified reinstatement procedure, we tested whether such reduced sexual reward induces reinstatement to drug-seeking. Together, these studies demonstrate that concurrent Meth-taking and sexual behavior enhances drugseeking behavior during extinction and reinstatement tests, indicative of enhanced vulnerability for addiction.

\section{METHODS AND MATERIALS}

Animals

Male Sprague Dawley rats (226-250 g; Charles River Laboratories, Wilmington, MA, USA) were housed in a climate-controlled facility under a $12-12 \mathrm{~h}$ reversed light-dark cycle. Male rats were socially housed throughout the studies in same-sex pairs. Food and water were available at all times in home cages, but not in the operant or mating chambers during behavioral testing sessions. Behavioral testing took place during the first half of the dark cycle. Female rats (Sprague Dawley, Charles River, 201-225 g) for sexual behavior testing were bilaterally ovariectomized under deep anesthesia (isoflurane gas; Piramal, Bethlehem, PA, USA) and received a subcutaneous silastic implant (Dow Corning Corp., Midland, MI, USA) containing $5 \%$ estradiol benzoate (Sigma-Aldrich, St. Louis, MO, USA) and 95\% cholesterol (Sigma-Aldrich). Sexual receptivity was induced by a subcutaneous (s.c.) injection of $500 \mu \mathrm{g}$ progesterone (Sigma-Aldrich) in $0.1 \mathrm{ml}$ sesame oil (Sigma-Aldrich) $4 \mathrm{~h}$ prior to testing. All experimental procedures were conducted in the dark phase under dim red lights and were approved by the Institutional Animal Care and Use Committee at the University of Mississippi Medical Center and conform to the guidelines outlined by the United States National Institutes of Health [26].

\section{Surgery}

Males received chronic indwelling catheters into the right jugular vein under deep anesthesia (2-3\% isoflurane gas) based on methods described in [27]. One end of a silastic catheter (Instech Laboratories, Plymouth Meeting, PA, USA) was inserted $3 \mathrm{~cm}$ into the right external jugular vein and secured with silk sutures (Look; Reading, PA, USA). The other end was tunneled subcutaneously to a midscapular incision, where it attached to a vascular access button (Instech) providing access for intravenous drug delivery. Aluminum caps (Instech) were used to protect the vascular access buttons, allowing animals to remain socially housed throughout the surgery recovery period and subsequent stages of the experiments. Animals were given carprofen ( $5 \mathrm{mg} / \mathrm{kg}$; s.c.) prior to surgery and once daily for the first $48 \mathrm{~h}$ after surgery. Catheters were flushed daily with $0.1 \mathrm{~mL}$ gentamicin $(5 \mathrm{mg} / \mathrm{mL})$ and $0.1 \mathrm{~mL}$ heparinized saline $(70 \mathrm{U} / \mathrm{mL})$ for one week after surgery. Thereafter, catheters were flushed before and after each selfadministration session with $0.1 \mathrm{~mL}$ heparinized saline to confirm and maintain catheter patency.

\section{Drug delivery}

Methamphetamine hydrochloride (Meth; Sigma-Aldrich) was dissolved in sterile saline either delivered intravenously (via jugular vein catheters; $0.04 \mathrm{mg} / \mathrm{kg}$ per infusion in $0.166 \mathrm{~mL}$; maximum of 25 infusions and $1 \mathrm{mg} / \mathrm{kg}$ total intake per session) or systemically ( $1 \mathrm{mg} / \mathrm{ml} / \mathrm{kg}$, i.p. for Meth-primed reinstatement). The dose per infusion had previously been demonstrated in dose-response testing to be maximally reinforcing [28]. The total Meth intake of $1 \mathrm{mg} / \mathrm{kg}$ dose was selected based on our previous studies showing that higher dosages, but not $1 \mathrm{mg} / \mathrm{kg}$, inhibited sexual behavior [19], and that passive administration of $1 \mathrm{mg} / \mathrm{kg}$ Meth concurrent with sex caused hypersexuality in male rats [19-21]. This dose is also well below neurotoxic dosage ranges [29].

\section{Behavioral testing}

Sexual behavior. Mating behavior was tested during the middle of the dark phase in the operant chamber or in a separate mating arena $(60 \times 45 \times 50 \mathrm{~cm})$. Males received a receptive female and mated until display of the first intromission following ejaculation. Parameters for sexual motivation and performance were recorded as follows: mount latency (ML; time from the introduction of the female until the first mount), intromission latency (IL; time from the introduction of the female until the first mount with vaginal penetration), ejaculation latency (EL; time from the first intromission until ejaculation), post ejaculation interval (PEl; time from ejaculation to first subsequent intromission), number of mounts (M), number of intromissions (IM), and copulation efficiency (number of intromissions divided by total mounts plus intromissions).

Meth self-administration. Self-administration and subsequent operant testing sessions were conducted in $30.5 \times 24 \times 21 \mathrm{~cm}$ operant chambers (Med Associates, St. Albans, VT) with two retractable levers and cue lights above both levers. Animals were not food deprived at any time and did not receive operant behavioral training prior to the onset of the studies. All selfadministration sessions started $3 \mathrm{~h}$ after onset of dark phase, and lasted for $3 \mathrm{~h}$ or until animals earned maximum drug infusions. Males self-administered Meth during 5 daily sessions on a fixedratio 1 (FR1) schedule of reinforcement. Each response on the active (left) lever resulted in delivery of $0.04 \mathrm{mg} / \mathrm{kg}$ Meth and illumination of a white light above the lever for $6 \mathrm{sec}$. Animals could earn a maximum of 25 infusions, for a total of $1 \mathrm{mg} / \mathrm{kg}$ Meth per session. Responses on the inactive (right) lever had no programmed consequences and were recorded as a measure of general locomotor activity. Both levers remained extended and lever responses were recorded during drug infusions. Responses on active and inactive levers, and numbers of infusions were recorded (Med Associates, MED-PC IV software).

Cue reactivity. A 30 min test during which responses on the active lever resulted in presentation of drug-associated cues (i.e., 6-second cue light and infusion pump sound), but no drug. Inactive lever responses had no programmed consequences. Levers remained extended during the entire session.

Extinction. Animals were subjected to 10-14 daily one-hour extinction sessions in which lever responses had no programmed consequences (Operant Extinction) or resulted in the presentation of drug-associated cues (6-second activation of cue light and pump sound; Cue Extinction). Levers remained extended during the entire session.

Sex-induced cue reinstatement (Sex Reinstatement). Males were allowed to mate to 1 ejaculation in a neutral cage (mating arena $60 \times 45 \times 50 \mathrm{~cm}$ Plexiglas cage with clean bedding) not associated with drug use and in a separate room from operant selfadministration boxes, immediately after which they were placed in the operant chamber for $1 \mathrm{~h}$, where active lever responding resulted in presentation of drug-associated cues (activation of cue light and pump sound for $6 \mathrm{sec}$ ). Levers remained extended during the entire session.

Cue Reinstatement. Males were placed in the operant chamber for $1 \mathrm{~h}$, where active lever responding resulted in presentation of drug-associated cues (activation of cue light and pump sound for $6 \mathrm{sec}$ ). Levers remained extended during the entire session. 
Meth-primed reinstatement (Meth Reinstatement). Males were injected with a bolus dose of Meth ( $1 \mathrm{mg} / \mathrm{ml} / \mathrm{kg}$, i.p.) $15 \mathrm{~min}$ prior to being placed in the operant chamber for $1 \mathrm{~h}$, where active lever responding resulted in presentation for drug-associated cues (activation of cue light and pump sound for $6 \mathrm{sec}$ ). Levers remained extended during the entire session. Habituation injections of saline ( $1 \mathrm{~mL} / \mathrm{kg}$, i.p.) were given $15 \mathrm{~min}$ prior to the extinction session preceding Meth Reinstatement.

Criteria for exclusion. All criteria for inclusion or exclusion of subjects and numbers of excluded subjects in all behavioral experiments are described in detail in the Supplementary Information.

\section{Experimental designs}

Self-administration, cue reactivity, extinction, and reinstatement. Concurrent and non-concurrent Meth/sex groups: prior to study onset in Experiments 1 and 2, all males mated during one prestudy mating session (in mating arena) and groups were matched for sexual behavior (Supporting Document Tables S1, S2). Subsequently, males either received sexual experience immediately after each Meth self-administration sessions (concurrent) or during the 5 days prior to the start of self-administration sessions (non-concurrent). All mating experiences took place in the mating arena, except for Experiment 1 concurrent group, which mated in the operant chamber).

In Experiment 1, animals (concurrent group $n=8$, nonconcurrent group $n=7$ ) underwent Meth self-administration for 5 consecutive days, 7 days of forced home-cage abstinence, cue reactivity, 10 daily operant extinction sessions, and Sex Reinstatement (see Fig. 1a for timeline).

In Experiment 2, animals (concurrent group $n=10$, nonconcurrent group $n=11$ ) underwent Meth self-administration for 5 days or to criteria (see below under Criteria for behavioral experiments), 14 daily cue extinction sessions (wherein cue reactivity was measured during the first $30 \mathrm{~min}$ of the first session), Sex Reinstatement, 3 additional cue extinction sessions, and Meth-primed reinstatement (see Fig. 2a for timeline).

In Experiment 3, parameters for Meth self-administration were identical to Experiments 1 and 2, except that the groups tested were non-concurrent $(n=5)$ and Meth only $(n=6)$. Meth-only animals remained sexually naive throughout the experiment and were returned to home cage after self-administration sessions. After Meth self-administration, animals underwent operant extinction for 10 days. Finally, the reinstatement phase consisted of two test sessions to test for vulnerability to relapse (Cue Reinstatement, Sex or Cue Reinstatement: non-concurrent males were mated prior to this second reinstatement test, while Methonly males instead were taken from their home cages and did not mate prior to the test) separated by additional operant extinction sessions (see Fig. 3a for timeline).

Statistical analyses. Statistical analyses were performed using SigmaPlot software (Systat Software, Inc., San Jose, CA), and all statistical information for reported effects and numbers of animals for each experiment are listed in Table 1.

During Meth self-administration and extinction in the selfadministration experiments, active lever responses, inactive lever responses, infusions earned, and mating parameters during repeated mating sessions were recorded and compared between and within groups using two-way repeated measures ANOVA with Holm-Sidak post-hoc pairwise comparisons. Active and inactive lever responses during reinstatement were recorded and compared using two-way ANOVA with the Holm-Sidak method for post-hoc pairwise comparisons.

Parameters for sexual behavior were compared using Student's $t$-tests or one-way ANOVA.

Self-administration experiments were conducted in several (2-3) cohorts. Each cohort included all experimental groups within the experiment, and all experimental effects (of concurrent Meth/sex) were replicated within each cohort. No statistical differences were detected between cohorts within groups, and data from the separate cohorts within groups were therefore combined for the final numbers of animals.

\section{RESULTS}

Experiment 1

Male rats underwent restricted-access Meth self-administration with concurrent sexual behavior in the operant chamber immediately following each self-administration session (Fig. 1a; Concurrent), or during daily sessions prior to Meth selfadministration in a separate mating arena (Fig. 1a; Nonconcurrent). Mating behavior was unaffected by Meth and sexual experience did not differ between groups (Table S1, Supporting Document). Operant behavior during the daily self-administration sessions did not differ between concurrent and non-concurrent groups as active and inactive lever responses were identical between groups (Fig. 1b). Animals in both groups earned maximum infusions during the final three sessions (Fig. 1c), and no group differences were detected in the time it took to obtain maximum infusions (Time to completion; Table S3, Supporting Document), although only the concurrent group significantly decreased the time to completion across sessions (Table S3, Supporting Document). Total Meth intake over all sessions did not differ between groups (Table S4, Supporting Document). Thus, any effect of concurrent Meth and sex exposure is not attributed to differences in drug taking. After self-administration, animals underwent 7 days of forced abstinence. Next, males were tested for operant cue reactivity during which concurrent and nonconcurrent groups did not differ in active responses for the drugassociated light, i.e., cue-seeking behavior (Fig. 1d). This test was followed by operant extinction sessions, during which both groups significantly reduced active responses across the 10 operant extinction sessions. However, the prior exposure to concurrent Meth and sex significantly enhanced drug-seeking during extinction (Fig. 1e): concurrent males responded significantly more on the active, but not inactive, lever in sessions $1,3,4$, 5 , and 7 , indicating an attenuation of extinction learning (Fig. 1e). Next, males mated in a neutral environment not associated with prior drug use, and were immediately tested for reinstatement of active responding for the drug-associated cues (Sex Reinstatement). Indeed, concurrent Meth/sex males significantly increased active responding (Fig. 1f) compared to the last day of extinction, while non-concurrent animals did not, and concurrent males responded significantly more on the active lever than nonconcurrent males during reinstatement. Responding on the inactive lever did not differ between groups in Sex Reinstatement (Table S5, Supporting Document).

\section{Experiment 2}

A second experiment was conducted to further test whether it was essential that drug taking and sexual behavior were experienced in the same physical environment. In addition, the abstinence period after the concurrent Meth and sex experience was omitted to investigate if inclusion of such abstinence potentially masked enhancement of Meth-associated cue seeking. Moreover, the second experiment tested if cue extinction training would be able to prevent effects of concurrent Meth and sex experience on drugassociated cue seeking. Finally, this experiment included a reinstatement test in which drug-seeking was triggered by passive administration of Meth. Thus, the second experiment had four key differences in the experimental design: (1) all mating behavior was conducted in a neutral environment not associated with drug use, (2) the abstinence period prior to extinction training was omitted, (3) extinction training included exposure to drug-associated cues upon active responses (Cue Extinction), and (4) Meth-primed 


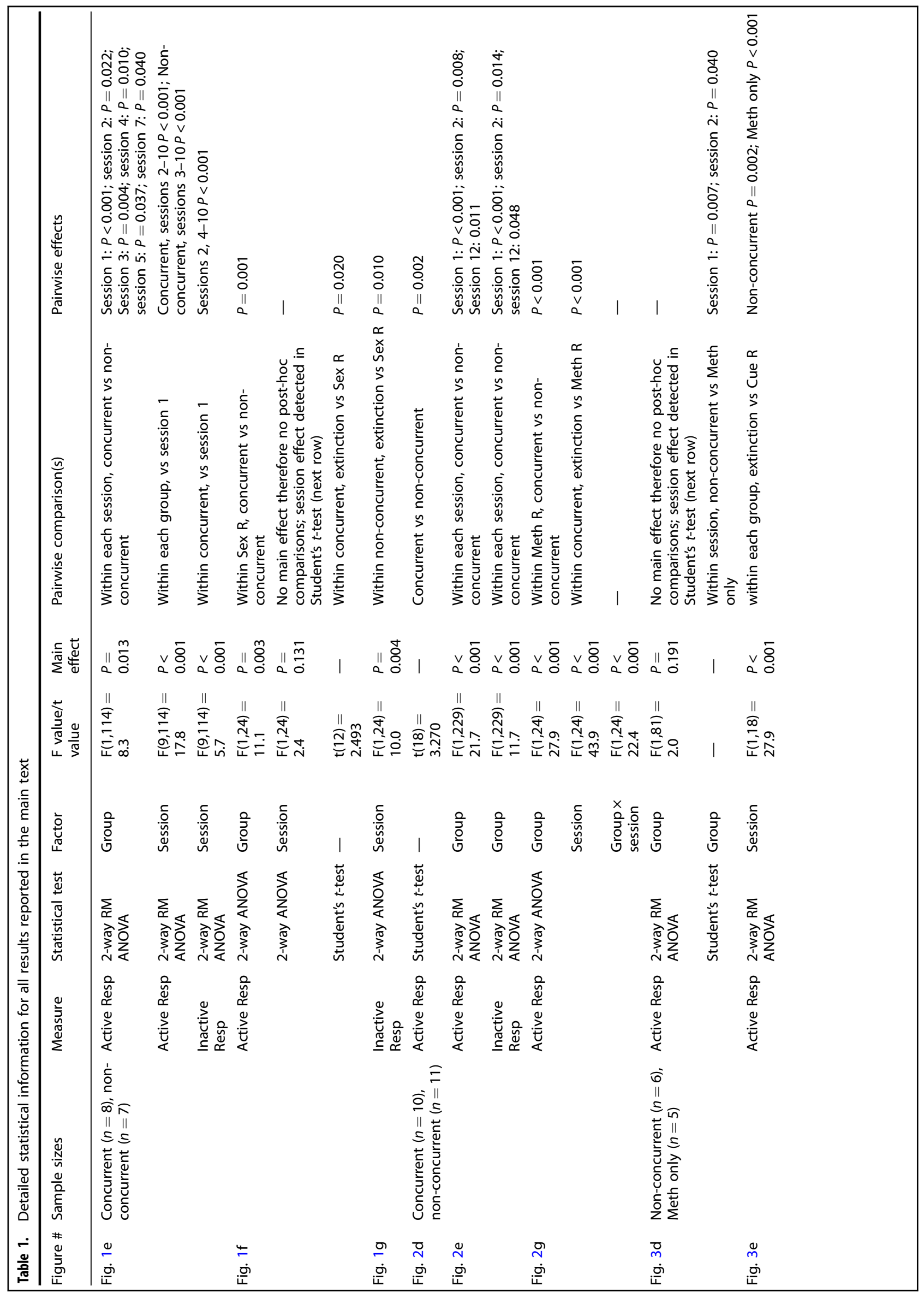




\begin{tabular}{|c|c|c|c|c|c|}
\hline & 5 sessions & 7 days & 1 session & 10 sessions & 1 session \\
\hline 5 sessions & $\begin{array}{l}\text { Meth SA } \\
+ \text { Sex }\end{array}$ & Abstinence & $\begin{array}{c}\text { Cue } \\
\text { Reactivity }\end{array}$ & $\begin{array}{l}\text { Operant } \\
\text { Extinction }\end{array}$ & $\begin{array}{c}\text { Sex } \\
\text { Reinstatement }\end{array}$ \\
\hline Sex & Meth SA & Abstinence & $\begin{array}{c}\text { Cue } \\
\text { Reactivity }\end{array}$ & $\begin{array}{l}\text { Operant } \\
\text { Extinction }\end{array}$ & $\begin{array}{c}\text { Sex } \\
\text { Reinstatement }\end{array}$ \\
\hline
\end{tabular}
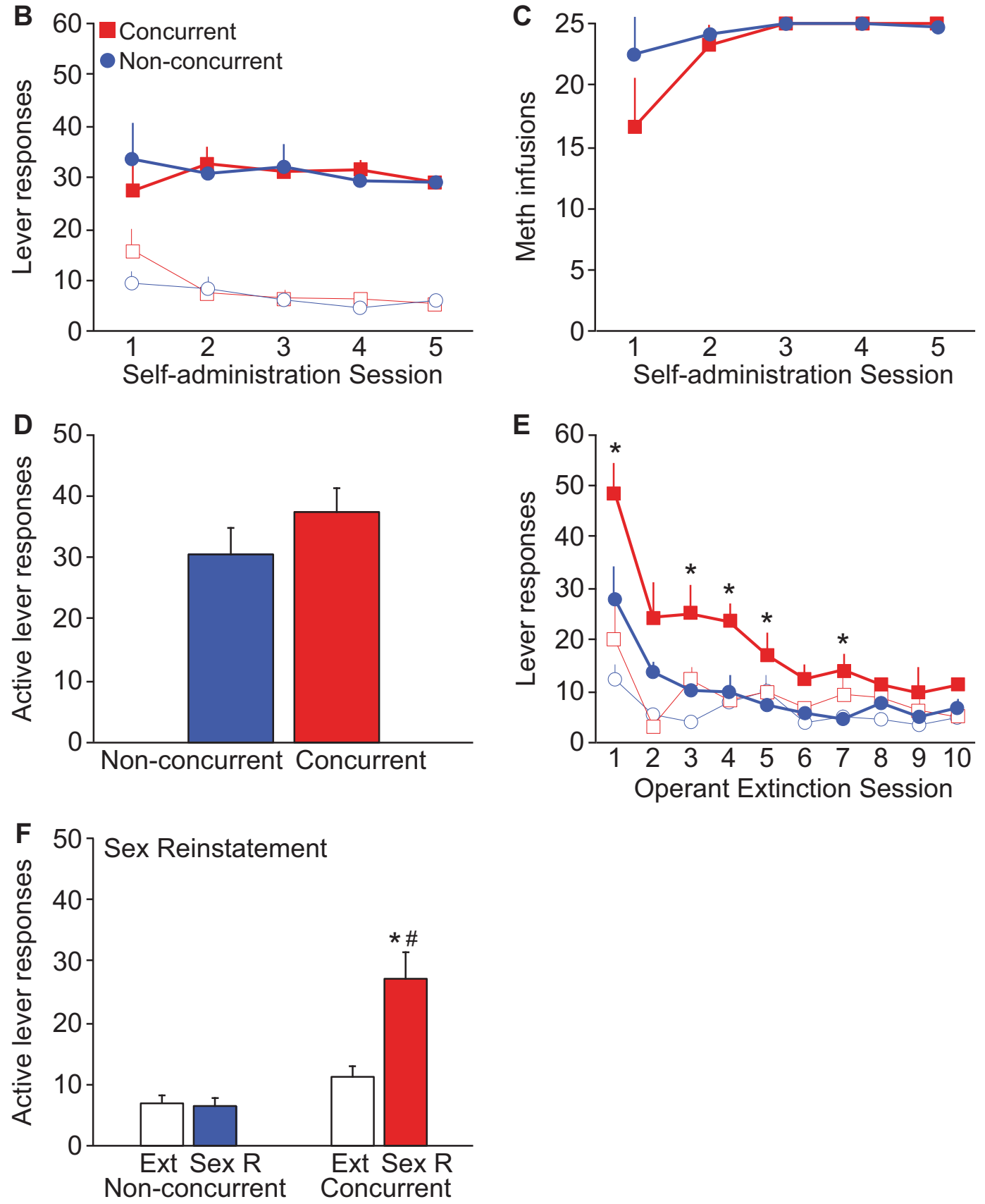

Fig. 1 Concurrent, but not non-concurrent, Meth self-administration and sex leads to increased drug-seeking behavior during operant extinction and sex reinstatement. a Experimental design. b Operant behavior (closed markers: active responses, open markers: inactive responses) and $\mathbf{c}$ Meth infusions earned during the final 5 sessions of Meth self-administration for the two experimental groups, $\mathbf{d}$ Active lever responses during 30 min cue reactivity test. e Concurrent animals displayed significantly increased drug-seeking behavior during sessions 1 , 3 , 4,5 , and 7 of operant extinction (indicated by *). f Active lever responses during Sex Reinstatement compared with the last day of extinction. The concurrent group showed significant reinstatement of active lever responding compared to the last day of extinction (indicated by *) and compared to the non-concurrent group (indicated by \#). All data are expressed as Mean + SEM. Sample sizes: concurrent $n=8$; nonconcurrent $n=7$ 
reinstatement was tested. Animals again received either concurrent or non-concurrent access to Meth self-administration and sex, but all mating occurred in a separate mating cage, hence not in the drug-taking environment (Fig. 2a). Mating behavior did not differ between groups (Table S2, Supporting Document). Moreover, groups did not differ in numbers of active nor inactive responses (Fig. 2b), Meth infusions (Fig. 2c), time to completion (Table S3, Supporting Document), nor total Meth intake (Table S4, Supporting Document). The first $30 \mathrm{~min}$ of the first day of extinction was analyzed as the cue reactivity test, comparable to the first experiment. Concurrent animals responded significantly more on the active lever compared to non-concurrent males during cue reactivity (Fig. $2 \mathrm{~d}$ ). Hence, the inclusion of an abstinence period in the first experiment possibly masked the effect of concurrent Meth/sex exposure by increasing drug-seeking behavior during the abstinence period in both groups consistent with the effects of "incubation of craving" [30]. During the remaining extinction training, concurrent males responded significantly more on the active lever (Fig. 2e) in sessions 1, 2, and 12 compared to nonconcurrent males. There were also group differences in inactive responding in these sessions (Fig. 2e). Next, sex-induced reinstatement of cue seeking was conducted as in experiment 1 , but failed to trigger reinstatement in either group (Fig. 2f), suggesting that cue extinction was able to overcome the effects of concurrent Meth and sex on Cue Reinstatement triggered by mating. Next, animals were tested for Meth-primed reinstatement of cue seeking (Meth Reinstatement). Concurrent Meth/sex experience significantly enhanced Meth-induced reinstatement compared to nonconcurrent and compared to last day of extinction (Fig. 2g). Specifically, concurrent, but not non-concurrent, animals significantly reinstated responding on the active lever (no group differences in inactive lever responding, Table S5, Supporting Document). In summary, these results further confirm the findings of Experiment 1 that concurrent Meth and sex exposure increases resistance to extinction and vulnerability to relapse to drugseeking. The results also show that mating-induced (Fig. 2f), but not Meth-induced reinstatement (Fig. 2g), was abolished by cue extinction training and that it is not essential for mating and Meth to be experienced in the same physical context. Since Experiments 1 and 2 differ in several key aspects of the experimental design, direct comparisons between the two studies are not valid. Therefore, direct comparisons between the physical contexts in which drug and sexual experience took place and between operant and cue extinction paradigms were further tested in Supplementary Experiment 1 (See Supporting Document).

\section{Experiment 3}

Results of experiments 1 and 2 showed that concurrent males have higher active lever presses during extinction and reinstatement sessions compared to non-concurrent males, suggesting that concurrent Meth and sex increases vulnerability for addiction. Experiment 3 tested an alternate explanation that prior sexual experience in the non-concurrent animals had protective effects, rendering these animals less vulnerable. Experiment 3 compared drug-seeking behavior in animals with non-concurrent sexual experience to that of animals with no sexual experience at all (Meth only; see Fig. 3a for timeline). There were no differences between groups during the Meth self-administration sessions in active and inactive lever responses (Fig. 3b), Meth infusions (Fig. 3c), total Meth intake (Table S4, Supporting Document), or time to completion (Table S3, Supporting Document), although non-concurrent, but not Meth only, males showed significantly reduced time to obtain the maximum infusions across sessions (Table S3, Supporting Document). The non-concurrent group responded significantly more during the first two extinction sessions (Fig. 3d), suggesting that prior sexual experience slightly enhanced rather than protected drug-seeking behavior. This finding is consistent with our previous reports showing effects of sexual experience and subsequent abstinence of sexual reward on CPP for psychostimulants [31]. During Cue Reinstatement test, groups showed significant reinstatement of active lever responding (Fig. 3e) compared to the previous extinction day, and groups were not different in numbers of active lever responses. In contrast, neither of the groups increased responding on the active nor inactive lever during the second Reinstatement test, during which non-concurrent males were tested for sex reinstatement and Meth-only males for cue reinstatement (Fig. 3f, Table S5, Supporting Document).

\section{DISCUSSION}

Together, these studies demonstrate that concurrent experience with Meth and sex significantly and reliably increased resistance to extinction and enhanced reinstatement of drug-seeking. Concurrent experience with Meth and sex also increases CPP for Meth alone [20], suggesting enhanced incentive salience of Meth and/or strengthened associations between Meth and drug-associated cues caused by Meth-taking in a socio-sexual context. These findings thus support the hypothesis that Meth exposure in the context of socio-sexual behavior promotes a shift from limited recreational voluntary use to drug addiction.

Elucidating the specific factors contributing to individual vulnerability to developing drug addiction is a critical step towards developing prevention and treatment strategies. The development of addiction is influenced by a variety of intrinsic factors, such as genetics and epigenetics, and extrinsic factors, including life experiences [32]. Animal research has provided evidence for a protective role of social and environmental enrichment [2, 33], while social isolation [2] or loss of sexual reward [4, 9] increase vulnerability for addiction. Recently it has been emphasized that preclinical addiction studies have failed to predict outcomes in human subjects, and that a major shortcoming of these studies is a lack of consideration of social influences in the experimental design [2], and studies henceforth will need to address this pitfall. Even with this increased awareness, the social influences on drug addiction vulnerability thus far have largely focused on effects of social isolation or providing social interactions to such isolated animals [34-37]. In addition, effects of social contact during drug taking behavior have been demonstrated. Specifically, cocaine selfadministration is enhanced in socially housed male or female rats if both members of the same-sex pair have simultaneous access to the drug in the same chamber using modified operant conditioning chambers, and in comparison to either socially isolated animals or socially housed animals that were paired with a drug abstinent partner [38-40]. The current study thus further extends these previous findings and provides a unique perspective in that it models the initial voluntary recreational drug taking in a sociosexual context with a sexual partner that was not part of the housing conditions. Findings indicate that a synergistic association of drug taking with socio-sexual activity significantly enhances vulnerability for addiction evidenced by increased drug-seeking behavior. These effects are not attributed to social isolation, as males were socially housed throughout the studies. In the current studies, all male rats were socially housed with a Meth-taking partner, but did not experience Meth-taking simultaneously with these housing partners and there were no differences in drug taking between groups. Hence, enhanced drug seeking in concurrent Meth and sex males was not caused by differences in drug exposure, as Meth intake was restricted to $1 \mathrm{mg} / \mathrm{kg} / \mathrm{day}$ and did not differ between groups. Future studies could explore effects of concurrent Meth and sex experience on subsequent drug taking using models of escalation of intake [41]. Additionally, progressive ratio protocols can be used to test effects on motivation for drug taking [42].

In contrast to the concurrent Meth and sex males, males that experienced Meth without the concurrent sexual experience 
A

\begin{tabular}{|c|c|c|c|c|c|}
\hline & 5 sessions & 14 sessions & 1 session & 3 sessions & 1 session \\
\hline 5 sessions & $\begin{array}{l}\text { Meth SA } \\
+ \text { Sex }\end{array}$ & $\begin{array}{c}\text { Cue } \\
\text { Extinction }\end{array}$ & $\begin{array}{c}\text { Sex } \\
\text { Reinstatement }\end{array}$ & $\begin{array}{c}\text { Cue } \\
\text { Extinction }\end{array}$ & $\begin{array}{c}\text { Meth } \\
\text { Reinstatement }\end{array}$ \\
\hline Sex & Meth SA & $\begin{array}{c}\text { Cue } \\
\text { Extinction }\end{array}$ & $\begin{array}{c}\text { Sex } \\
\text { Reinstatement }\end{array}$ & $\begin{array}{c}\text { Cue } \\
\text { Extinction }\end{array}$ & $\begin{array}{c}\text { Meth } \\
\text { Reinstatement }\end{array}$ \\
\hline
\end{tabular}

B

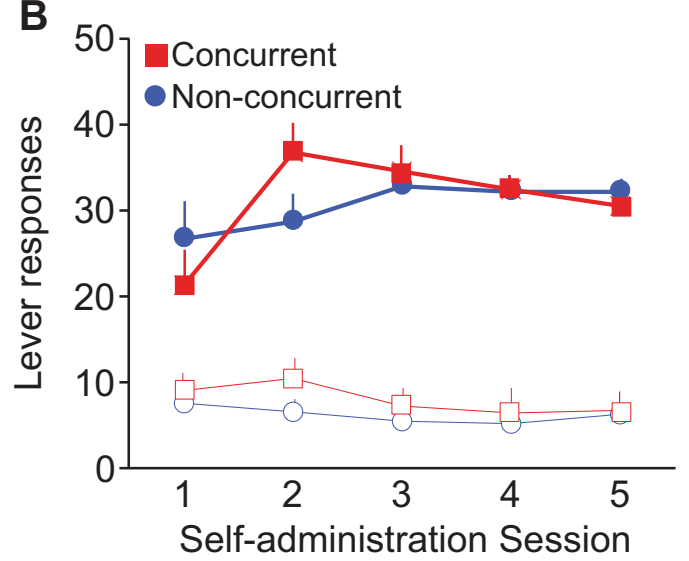

C

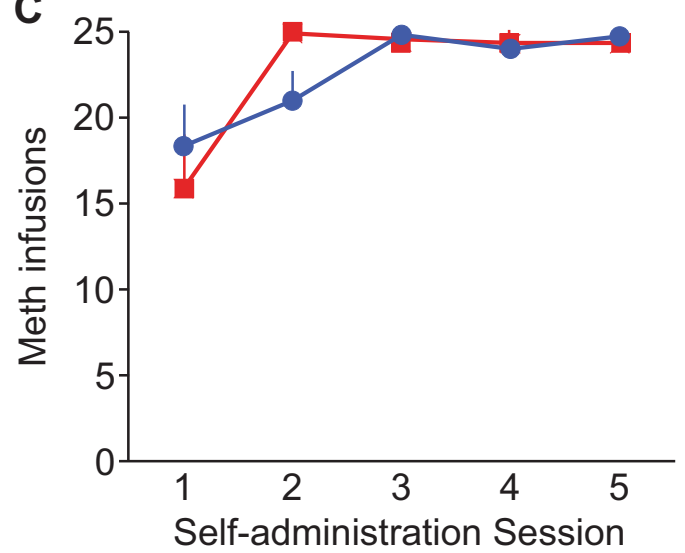

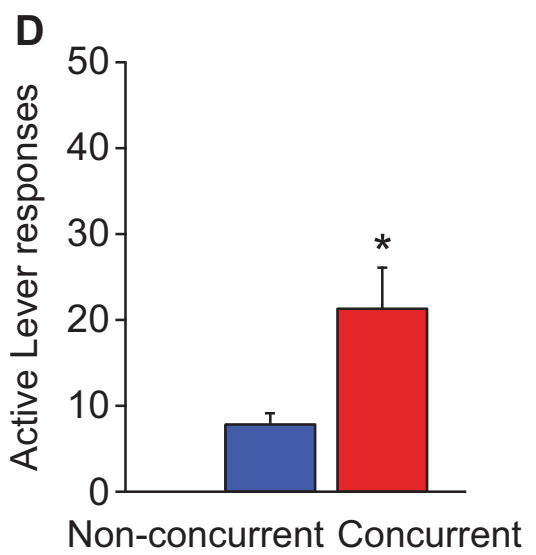

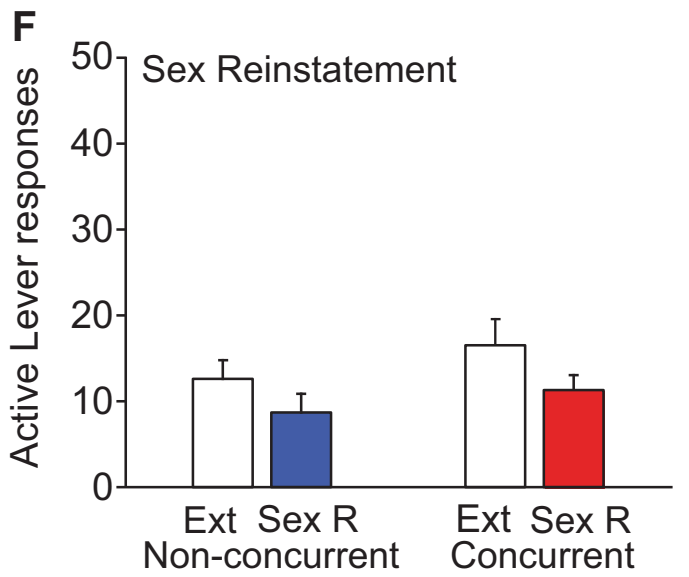

E 50
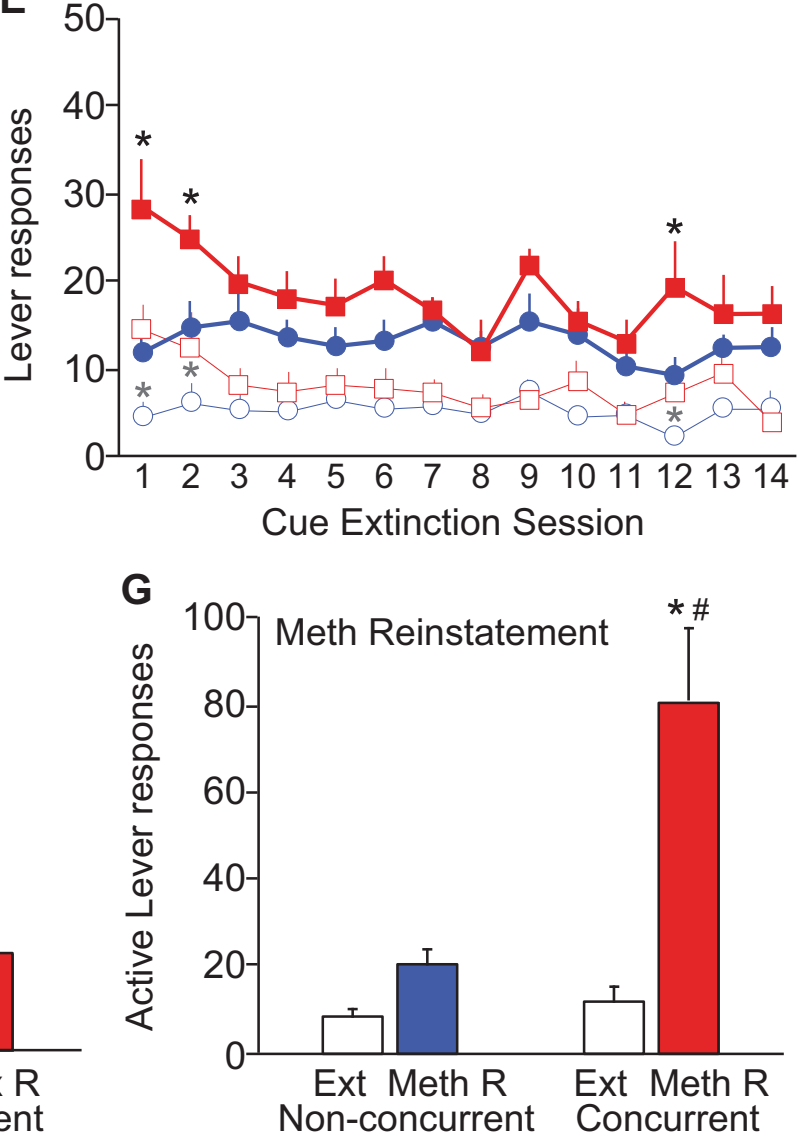

Fig. 2 Concurrent Meth self-administration and sex leads to increased drug-seeking behavior during cue extinction and Meth-primed reinstatement. a Experimental design. b Operant behavior (closed markers: active responses, open markers: inactive responses) and c Meth infusions earned during the final 5 sessions Meth self-administration. d Concurrent animals displayed significantly increased drug-seeking behavior (active lever responses) during the first $30 \mathrm{~min}$ of the first cue extinction session (indicated by ${ }^{*}$ ). e Concurrent animals displayed significantly increased drug-seeking behavior during sessions 1, 2, and 12 of cue extinction (indicated by *). $\mathbf{f}$ Sex Reinstatement and $\mathbf{g}$ Meth Reinstatement active lever responding. The concurrent group displayed significant reinstatement vs the last day of extinction (indicated by ${ }^{*}$ ) and vs the non-concurrent group during Meth Reinstatement (indicated by \#). All data are expressed as Mean + SEM. Sample sizes: concurrent $n=10$; non-concurrent $n=11$ 
A

\begin{tabular}{c|c|c|c|c|c|}
\multicolumn{1}{c}{} & \multicolumn{2}{c}{5 sessions } & 10 sessions & \multicolumn{1}{c}{1 session } & \multicolumn{2}{c|}{2 sessions } & 1 session \\
\cline { 2 - 3 } \multicolumn{1}{c|}{5 sessions } & Meth SA & $\begin{array}{c}\text { Operant } \\
\text { Extinction }\end{array}$ & $\begin{array}{c}\text { Cue } \\
\text { Reinstatement }\end{array}$ & $\begin{array}{c}\text { Operant } \\
\text { Extinction }\end{array}$ & $\begin{array}{c}\text { Cue } \\
\text { Reinstatement }\end{array}$ \\
\hline Sex & Meth SA & $\begin{array}{c}\text { Operant } \\
\text { Extinction }\end{array}$ & $\begin{array}{c}\text { Cue } \\
\text { Reinstatement }\end{array}$ & $\begin{array}{c}\text { Operant } \\
\text { Extinction }\end{array}$ & $\begin{array}{c}\text { Sex } \\
\text { Reinstatement }\end{array}$ \\
\hline
\end{tabular}

B

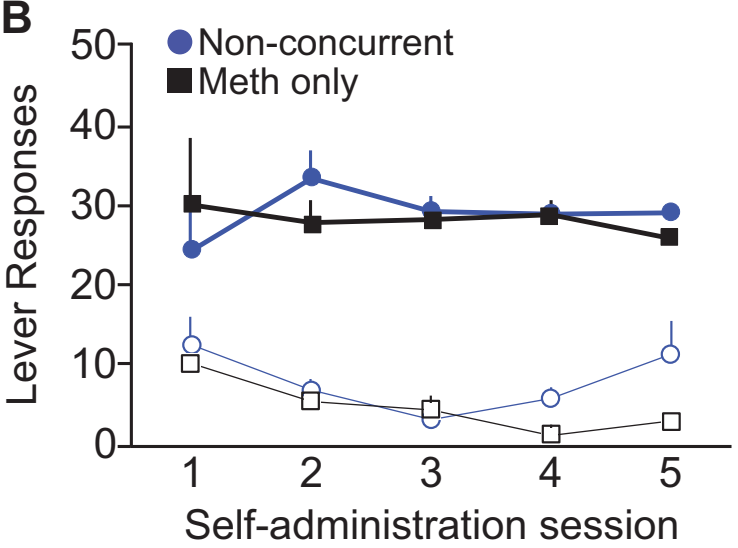

D

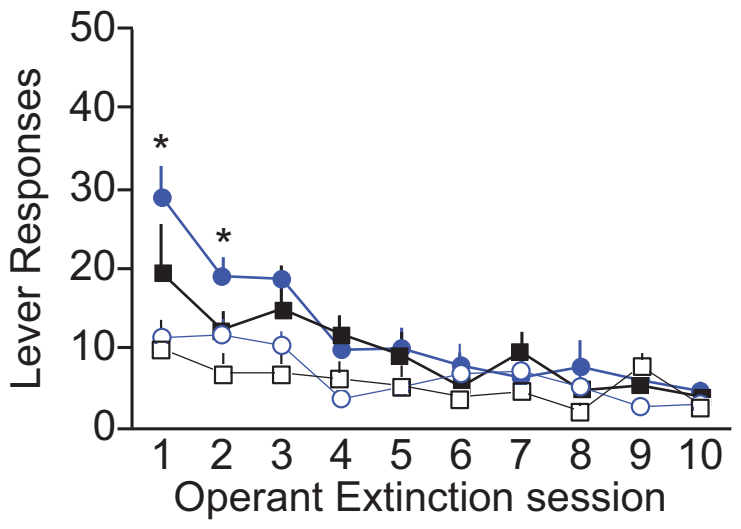

C

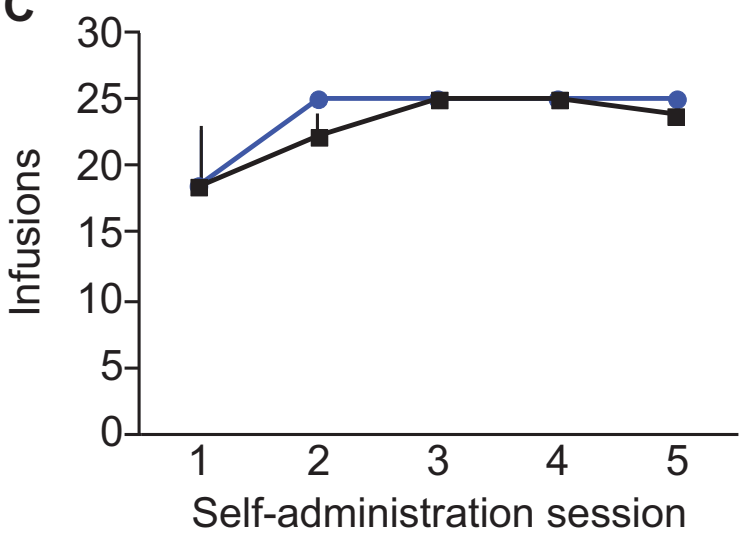

E

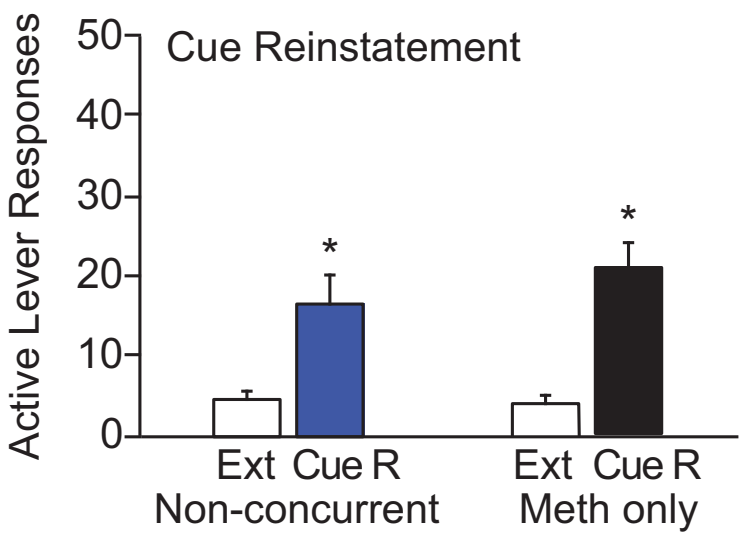

$\mathbf{F}$

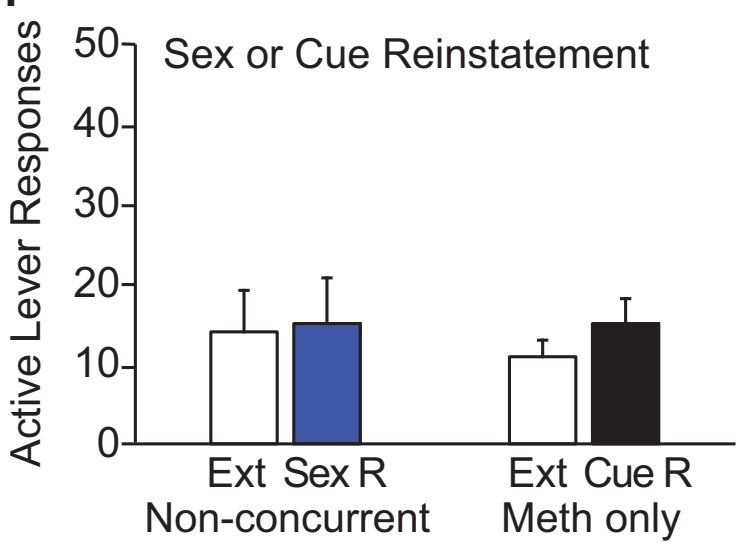

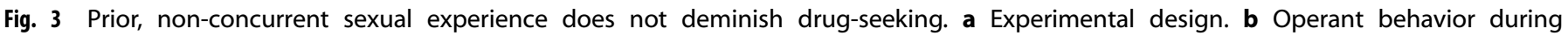

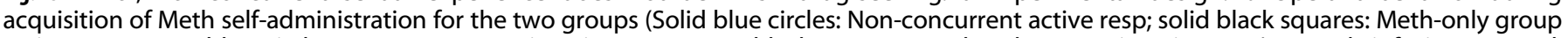

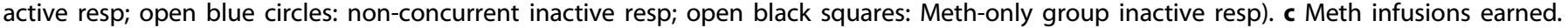

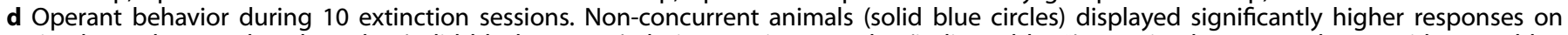
active lever then Meth only males (solid black squares) during sessions 1 and 2 (indicated by *). Inactive levers are shown with open blue

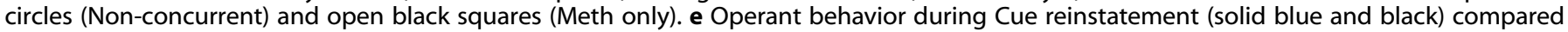

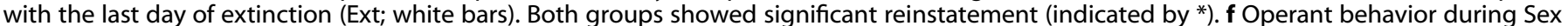

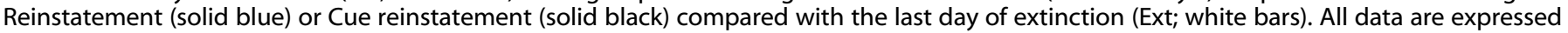
as Mean + SEM. Sample sizes: non-concurrent $n=6$, Meth only $n=5$ 
showed little to no reinstatement. This lack of reinstatement or reduced responding in non-concurrent males is consistent with our supposition that limited Meth exposure alone is less effective to increase propensity to relapse. Previous publications documenting Cue Reinstatement typically use longer durations of Meth exposure and higher dosages per session (intake of $2-5 \mathrm{mg} / \mathrm{kg} /$ session during 10-15 sessions) [30,43,44]. Of note, concurrent Meth and sex males showed robust mating-induced reinstatement, consistent with the previous findings that sex behavior does not induce CPP in these males [20], and suggesting a loss of reward for mating when experienced in absence of drug. Hence, the loss of reward normally associated with sex may further promote reinstatement of drug-seeking behavior, consistent with anecdotal reports in human users $[18,45]$. Interestingly, cue extinction, which breaks the contingency between the drug (unconditioned) and conditioned stimuli, was sufficient to overcome the effects of concurrent Meth and sex experience on mating-induced reinstatement. Moreover, findings from Experiment 3 suggest that lack of cue-, sex-, or Meth reinstatement in non-concurrent males was not due to prior sexual experience having protective effects. Instead, a slight sensitization effect was noted with enhanced lever responding during the first extinction sessions. Nonetheless, protective effects in non-concurrent males may have been present from the mating behavior immediately prior to the Sex Reinstatement (Experiment 1), as this group did not show reinstatement induced by mating behavior. In both sexually naïve and experienced males, sexual behavior induces CPP [46], indicative of sex-associated reward. Thus, the rewarding aspects of sexual behavior may have reduced drug-seeking behavior in the reinstatement tests in non-concurrent males. An alternative interpretation of the lack of sex reinstatement in nonconcurrent males is due to a rapid extinguishing of drug-paired cue-associations, for example in Experiment 1 during the cue reactivity test prior to operant extinction. Indeed, in Experiment 3, the Meth-only group failed to show cue reinstatement during the second cue-reinstatement test, possibly due to extinction during the first cue-reinstatement test. Together these findings suggest that a single session with non-contingent cues is sufficient to extinguish subsequent drug-seeking in non-concurrent and Methonly males. In contrast, concurrent males are more resistant to rapid extinguishing of drug-paired cue-associations, further supporting the hypothesis and findings of increased vulnerability for addiction.

Finally, it is of note that the effects of concurrent Meth and sex in the current studies were independent of the physical environment in which sexual behavior was experienced and thus independent of a physical contextual association between sex and drug environments. We therefore hypothesize that this is a synergistic, rather than an additive, effect that is based on a memory of the Meth/sex association. Such association may instead be dependent on the intrinsic context of mating at times that levels of Meth and amphetamines are elevated in the brain [47], resulting in enhanced incentive salience of Meth.

A limitation of the current study is the exclusion of female subjects, though comorbidity of compulsive sexual behavior and drug addiction occurs in both men and women and is independent of sexual orientation [11, 12]. However, female rats only display sexual receptivity once in every four days during behavioral estrus [48], and thus do not simulate human sexual behavior in which estrous cycle phase is not obligatory to sexual motivation and behavior. From the current studies, it is unclear if copulation is necessary for the enhancement of addiction by concurrent Meth/sex, or whether social interaction would also be sufficient. This warrants future studies to determine if drug use concurrent with social interaction, in males and females, will produce the vulnerable phenotype observed in the present studies. Finally, future studies may investigate if the effects reported here are specific to socio-sexual behaviors versus other natural rewards. Even though comparisons with food-related rewards may be problematic due to the anorexic effects of Meth [49], it may be interesting to explore if exposure to other natural rewards (i.e., sucrose, wheel running) immediately following Meth administration also induces enhancing effects on drug-seeking behaviors. Investigation of a choice between drug and natural reward seeking has demonstrated that concurrent sucrose exposure during Meth self-administration or reinstatement tests decreases Meth intake and drug-seeking [50]. Thus, providing the choice for an alternative reinforcer may decrease drug seeking and taking. In the current experiments, sexual behavior was not presented as a choice or alternative for Meth seeking behavior, but instead was presented after drug taking had been completed, and thus occurred within the same intrinsic context. We have previously demonstrated using CPP procedures that such concurrent Meth and sex experience causes an enhanced CPP compared to Meth or sex alone [20].

The underlying neural mechanisms by which concurrent drug and sex experience influences subsequent drug-seeking behaviors are unclear. Our laboratory has previously shown that concurrent Meth and sexual behavior co-activate neurons in the mesocorticolimbic system, specifically in the anterior cingulate area (ACA) of the medial prefrontal cortex (mPFC), basolateral amygdala, and nucleus accumbens core and shell [51]. Moreover, concurrent Meth and sexual behavior increases baseline neuronal activity in CaMKII-expressing projection neurons in the ACA, indicating neuroplasticity induced by concurrent Meth and sex [21]. Future studies will investigate the role that co-activation of these neurons during concurrent Meth and sex plays in enhanced drug-seeking behavior in extinction or reinstatement. It has been demonstrated in both drug- and food-seeking studies that neurons encoding extinction memories versus those encoding reward memories reside in the $\mathrm{mPFC}$ (ventral $\mathrm{mPFC}$ and dorsal $\mathrm{mPFC}$, respectively) $[52,53]$, and that expression of these memories is abolished when these neurons are selectively lesioned [54, 55]. It is likely, therefore, that distinct neuronal ensembles are implicated in the failure to suppress behavior during the initial phases of extinction learning versus during the expression of drug-associated memory in reinstatement in the concurrent Meth/sex animals.

In conclusion, these studies demonstrate using male rats that Meth-taking in a socio-sexual context enhances drug-seeking behavior during extinction and reinstatement, suggesting that the memory for an association between drug and socio-sexual behavior may influence the shift to habit formation. These studies thus introduce a preclinical model to study the neural substrates of enhanced vulnerability to drug addiction in this context, potentially leading to a better understanding of the pathology underlying both substance abuse and hypersexuality.

\section{ACKNOWLEDGEMENTS}

The authors thank Drs. T. Celeste Napier, Amanda Persons, and Kevin Freeman for assistance with development of self-administration protocols, and medical student Sallie Lin for help with animal handling.

\section{AUTHOR CONTRIBUTION}

L.B.K., L.N.B., and L.M.C. designed the experiments, L.B.K., L.N.B., and B.M.D. recorded and analyzed data, and L.B.K., L.N.B., and L.M.C. prepared the manuscript.

\section{FUNDING}

Funding was provided by grants from Institutional Research Support Program at University of Mississippi Medical Center.

DATA AVAILABILITY

All full datasets are available upon request. 


\section{ADDITIONAL INFORMATION}

Supplementary Information accompanies this paper at (https://doi.org/10.1038/ s41386-018-0235-1).

Competing interests: The authors declare no competing interests.

Publisher's note: Springer Nature remains neutral with regard to jurisdictional claims in published maps and institutional affiliations.

\section{REFERENCES}

1. Koob GF, Volkow ND. Neurobiology of addiction: a neurocircuitry analysis. Lancet Psychiatry. 2016;3:760-73.

2. Heilig M, Epstein DH, Nader MA, Shaham Y. Time to connect: bringing social context into addiction neuroscience. Nat Rev Neurosci. 2016;17:592-9.

3. Frohmader KS, Pitchers KK, Balfour ME, Coolen LM. Mixing pleasures: review of the effects of drugs on sex behavior in humans and animal models. Horm Behav. 2010;58:149-62.

4. Beloate LN, Coolen LM. Influences of social reward experience on behavioral responses to drugs of abuse: review of shared and divergent neural plasticity mechanisms for sexual reward and drugs of abuse. Neurosci Biobehav Rev. 2017;83:356-72.

5. Frascella J, Potenza MN, Brown LL, Childress AR. Shared brain vulnerabilities open the way for nonsubstance addictions: carving addiction at a new joint? Ann N Y Acad Sci. 2010;1187:294-315.

6. Probst CC, van Eimeren T. The functional anatomy of impulse control disorders. Curr Neurol Neurosci Rep. 2013;13:386.

7. Banca $P$, Harrison NA, Voon V. Compulsivity across the pathological misuse of drug and non-drug rewards. Front Behav Neurosci. 2016;10:154.

8. Kraus SW, Voon V, Potenza MN. Should compulsive sexual behavior be considered an addiction? Addiction . 2016;111:2097-106.

9. Beloate LN, Coolen LM. Effects of sexual experience on psychostimulant- and opiate-induced behavior and neural plasticity in the mesocorticolimbic pathway. Int Rev Neurobiol. 2018;140:249-70.

10. Kuiper LB, Coolen LM. Compulsive sexual behavior in humans and preclinical models. Curr Sexual health Rep. 2018;10:124-31.

11. Johnson MW, Johnson PS, Herrmann ES, Sweeney MM. Delay and probability discounting of sexual and monetary outcomes in individuals with cocaine use disorders and matched controls. PloS ONE. 2015;10:e0128641.

12. Mausbach BT, Semple SJ, Strathdee SA, Patterson TL. Predictors of safer sex intentions and protected sex among heterosexual HIV-negative methamphetamine users: an expanded model of the Theory of Planned Behavior. AIDS Care. 2009;21:17-24.

13. Volkow ND, Wang G-J, Fowler JS, Telang F, Jayne M, Wong C. Stimulant-induced enhanced sexual desire as a potential contributing factor in HIV transmission. Am J Psychiatry. 2007;164:157-60.

14. Tull MT, Gratz KL. Major depression and risky sexual behavior among substance dependent patients: the moderating roles of distress tolerance and gender. Cogn Ther Res. 2013;37:483-97.

15. Hartzler B, Beadnell B, Calsyn DA. Use of sexual risk assessment and feedback at intake to promote counselor awareness of subsequent client risk behavior during early treatment. Subst Use Misuse. 2014;49:1270-7.

16. Wray TB, Celio MA, Kahler CW, Barnett NP, Mastroleo NR, Operario D, et al. Daily co-occurrence of alcohol use and high-risk sexual behavior among heterosexual, heavy drinking emergency department patients. Drug Alcohol Depend. 2015;152:109-15.

17. Green Al, Halkitis PN. Crystal methamphetamine and sexual sociality in an urban gay subculture: an elective affinity. Culture Health Sex. 2006;8:317-33.

18. Lyons T, Chandra G, Goldstein J, Ostrow DG. Breaking the bond between stimulant use and risky sex: a qualitative study. Subst Abus. 2010;31:224-30.

19. Frohmader KS, Bateman KL, Lehman MN, Coolen LM. Effects of methamphetamine on sexual performance and compulsive sex behavior in male rats. Psychopharmacol (Berl). 2010;212:93-104.

20. Frohmader KS, Lehman MN, Laviolette SR, Coolen LM. Concurrent exposure to methamphetamine and sexual behavior enhances subsequent drug reward and causes compulsive sexual behavior in male rats. J Neurosci. 2011;31:16473-82.

21. Kuiper LB, Frohmader KS, Coolen LM. Maladaptive sexual behavior following concurrent methamphetamine and sexual experience in male rats is associated with altered neural activity in frontal cortex. Neuropsychopharmacology. 2017:42:2011-20.

22. Napier TC, Herrold AA, de Wit $\mathrm{H}$. Using conditioned place preference to identify relapse prevention medications. Neurosci Biobehav Rev. 2013;37:2081-6.

23. Bossert JM, Marchant NJ, Calu DJ, Shaham Y. The reinstatement model of drug relapse: recent neurobiological findings, emerging research topics, and translational research. Psychopharmacology (Berl).2013;229:453-76.
24. Cho AK, Melega WP, Kuczenski R, Segal DS. Relevance of pharmacokinetic parameters in animal models of methamphetamine abuse. Synapse 2001;39:161-6.

25. Venniro M, Caprioli D, Shaham Y. Animal models of drug relapse and craving: from drug priming-induced reinstatement to incubation of craving after voluntary abstinence. Prog Brain Res. 2016;224:25-52.

26. Council NR. Guide for the Care and Use of Laboratory Animals. Eighth Edition. Washington, DC: The National Academies Press; 2011.

27. Thomsen M, Caine SB. Chronic intravenous drug self-administration in rats and mice. Curr Protoc Neurosci. 2005;9:Unit9 20.

28. Snider SE, Hendrick ES, Beardsley PM. Glial cell modulators attenuate methamphetamine self-administration in the rat. Eur J Pharmacol. 2013;701:124-30.

29. Davidson C, Gow AJ, Lee TH, Ellinwood EH. Methamphetamine neurotoxicity: necrotic and apoptotic mechanisms and relevance to human abuse and treatment. Brain Res Brain Res Rev. 2001;36:1-22.

30. Venniro M, Zhang M, Shaham Y, Caprioli D. Incubation of methamphetamine but not heroin craving after voluntary abstinence in male and female rats. Neuropsychopharmacology . 2017;42:1126-35.

31. Beloate LN, Omrani A, Adan RA, Webb IC, Coolen LM Ventral Tegmental Area Dopamine Cell Activation during Male Rat Sexual Behavior Regulates Neuroplasticity and d-Amphetamine Cross-Sensitization following Sex Abstinence. J Neurosci 2016;36:9949-61.

32. Egervari G, Ciccocioppo R, Jentsch JD, Hurd YL. Shaping vulnerability to addiction - the contribution of behavior, neural circuits and molecular mechanisms. Neurosci Biobehav Rev. 2018;85:117-25.

33. Darna M, Beckmann JS, Gipson CD, Bardo MT, Dwoskin LP. Effect of environmental enrichment on dopamine and serotonin transporters and glutamate neurotransmission in medial prefrontal and orbitofrontal cortex. Brain Res. 2015;1599:115-25.

34. Chauvet C, Goldberg SR, Jaber M, Solinas M. Effects of environmental enrichment on the incubation of cocaine craving. Neuropharmacology . 2012;63:635-41.

35. Chauvet $C$, Lardeux V, Jaber M, Solinas M. Brain regions associated with the reversal of cocaine conditioned place preference by environmental enrichment. J Neurosci. 2011;184:88-96.

36. Zernig G, Pinheiro BS. Dyadic social interaction inhibits cocaine-conditioned place preference and the associated activation of the accumbens corridor. Behav Pharmacol. 2015;26:580-94.

37. Lesscher HM, Spoelder M, Rotte MD, Janssen MJ, Hesseling P, Lozeman-van't Klooster JG, et al. Early social isolation augments alcohol consumption in rats. Behav Pharmacol. 2015:26:673-80.

38. Smith MA. Peer influences on drug self-administration: social facilitation and social inhibition of cocaine intake in male rats. Psychopharmacol (Berl). 2012;224:81-90.

39. Thiel KJ, Okun AC, Neisewander JL. Social reward-conditioned place preference: a model revealing an interaction between cocaine and social context rewards in rats. Drug Alcohol Depend. 2008;96:202-12.

40. Robinson AM, Fronk GE, Zhang H, Tonidandel S, Smith MA. The effects of social contact on cocaine intake in female rats. Drug Alcohol Depend. 2017;177: $48-53$.

41. Anker JJ, Baron TR, Zlebnik NE, Carroll ME. Escalation of methamphetamine selfadministration in adolescent and adult rats. Drug Alcohol Depend. 2012;124:149-53.

42. Richardson NR, Roberts DC. Progressive ratio schedules in drug selfadministration studies in rats: a method to evaluate reinforcing efficacy. J Neurosci Methods. 1996;66:1-11.

43. Lewis CR, Staudinger K, Scheck L, Olive MF. The effects of maternal separation on adult methamphetamine self-administration, extinction, reinstatement, and MeCP2 immunoreactivity in the nucleus accumbens. Front Psychiatry. 2013; 4:55.

44. Parsegian A, See RE. Dysregulation of dopamine and glutamate release in the prefrontal cortex and nucleus accumbens following methamphetamine selfadministration and during reinstatement in rats. Neuropsychopharmacology . 2014;39:811-22.

45. Lorvick J, Bourgois P, Wenger LD, Arreola SG, Lutnick A, Wechsberg WM, et al. Sexual pleasure and sexual risk among women who use methamphetamine: a mixed methods study. Int J Drug Policy. 2012;23:385-92.

46. Tenk CM, Wilson H, Zhang Q, Pitchers KK, Coolen LM. Sexual reward in male rats: effects of sexual experience on conditioned place preferences associated with ejaculation and intromissions. Horm Behav. 2009;55:93-7.

47. Riviere GJ, Gentry WB, Owens SM. Disposition of methamphetamine and its metabolite amphetamine in brain and other tissues in rats after intravenous administration. J Pharmacol Exp Ther. 2000;292:1042-7.

48. Veening JG, Coolen LM, Gerrits PO. Neural mechanisms of female sexual behavior in the rat; comparison with male ejaculatory control. Pharmacol Biochem Behav. 2014;121:16-30. 
Drug-taking in a socio-sexual context enhances vulnerability for...

LB Kuiper et al.

49. Bittner SE, Wagner GC, Aigner TG, Seiden LS. Effects of a high-dose treatment of methamphetamine on caudate dopamine and anorexia in rats. Pharmacol Biochem Behav. 1981;14:481-6.

50. Ping A, Kruzich PJ. Concurrent access to sucrose pellets decreases methamphetamineseeking behavior in Lewis rats. Pharmacol Biochem Behav. 2008;90:492-6.

51. Frohmader KS, Wiskerke J, Wise RA, Lehman MN, Coolen LM. Methamphetamine acts on subpopulations of neurons regulating sexual behavior in male rats. J Neurosci. 2010;166:771-84.

52. Pinto L, Dan Y. Cell-type-specific activity in prefrontal cortex during goal-directed behavior. Neuron 2015;87:437-50.
53. Moorman DE, Aston-Jones G. Prefrontal neurons encode context-based response execution and inhibition in reward seeking and extinction. Proc Natl Acad Sci USA. 2015;112:9472-7.

54. Whitaker LR, Warren $\mathrm{BL}$, Venniro $\mathrm{M}$, Harte $\mathrm{TC}$, McPherson $\mathrm{KB}$, Beidel J, et al. Bidirectional modulation of intrinsic excitability in rat prelimbic cortex neuronal ensembles and non-ensembles after operant learning. J Neurosci. 2017;37:8845-56.

55. Warren BL, Mendoza MP, Cruz FC, Leao RM, Caprioli D, Rubio FJ, et al. Distinct Fos-expressing neuronal ensembles in the ventromedial prefrontal cortex mediate food reward and extinction memories. J Neurosci. 2016;36:6691-703. 